\title{
Theoretical predictions for charm and bottom production at the LHC
}

\author{
Matteo Cacciari, ${ }^{a, b}$ Stefano Frixione,,$^{c, d, 1}$ Nicolas Houdeau, ${ }^{a}$ \\ Michelangelo L. Mangano, ${ }^{c}$ Paolo Nason, ${ }^{c, e, 2}$ and Giovanni Ridolfi ${ }^{f}$ \\ ${ }^{a}$ LPTHE, UPMC Université Paris 6 and CNRS UMR 7589, \\ Paris, France \\ ${ }^{b}$ Université Paris Diderot - Paris 7, \\ Paris, France \\ ${ }^{c}$ PH Department, TH Unit, CERN, \\ CH-1211 Geneva 23, Switzerland \\ ${ }^{d}$ Institut de Théorie des Phénomènes Physiques, EPFL, \\ CH-1015 Lausanne, Switzerland

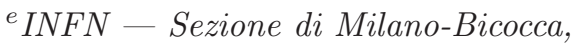 \\ Piazza della Scienza 3, 20126 Milan, Italy \\ ${ }^{f}$ Dipartimento di Fisica, Università di Genova, and INFN - Sezione di Genova, \\ Via Dodecaneso 33, I-16146 Genoa, Italy \\ E-mail: matteo.cacciari@lpthe.jussieu.fr, stefano.frixione@cern.ch, \\ nicolas.houdeau@lpthe.jussieu.fr, michelangelo.mangano@cern.ch, \\ paolo.nason@mib.infn.it, giovanni.ridolfi@ge.infn.it
}

ABSTRACT: We present predictions for a variety of single-inclusive observables that stem from the production of charm and bottom quark pairs at the $7 \mathrm{TeV}$ LHC. They are obtained within the FONLL semi-analytical framework, and with two "Monte Carlo + NLO" approaches, MC@NLO and POWHEG. Results are given for final states and acceptance cuts that are as close as possible to those used by experimental collaborations and, where feasible, are compared to LHC data.

KEYwords: QCD Phenomenology, Hadronic Colliders

\footnotetext{
${ }^{1}$ On leave of absence from INFN, Sezione di Genova, Genoa, Italy.

${ }^{2}$ On leave of absence from INFN, Sezione di Milano Bicocca, Milan, Italy.
} 


\section{Contents}

1 Introduction 1

2 Description of the theoretical frameworks 2

2.1 FONLL 2

2.1.1 Non-perturbative fragmentation 3

2.1.2 Theoretical uncertainties 4

2.2 NLO+PS approaches 5

2.2.1 MC@NLO 6

$\begin{array}{lll}2.2 .2 & \text { POWHEG } & 7\end{array}$

3 Numerical predictions and comparisons $\quad 8$

3.1 Open charm production 8

3.2 Open bottom production from inclusive and fully-reconstructed $H_{b} \rightarrow D+X$ $\begin{array}{ll}\text { decays } & 10\end{array}$

3.3 Open bottom production from inclusive $H_{b} \rightarrow J / \psi$ and $H_{b} \rightarrow \psi(2 S)$ decays 12

4 Open charm and bottom production from inclusive semileptonic decays 15

$\begin{array}{lll}5 & \text { Conclusions } & 17\end{array}$

\section{Introduction}

Measurements of charm and bottom production are among the most interesting studies of QCD dynamics that have emerged from the analysis of the first LHC data at $7 \mathrm{TeV}$ centreof-mass energy. The large total cross sections, expected to be of the order of $5 \mathrm{mb}$ (charm) and $250 \mu \mathrm{b}$ (bottom), have provided abundant data samples already with the $\sim 50 \mathrm{pb}^{-1}$ collected in 2010. A precise knowledge of charm and bottom quark cross sections and distributions is important in order to assess the accuracy of QCD calculations, but also as a test of theoretical predictions susceptible of being used to estimate backgrounds to new physics searches. This is all the more true since, in the past, early experimental measurements of bottom production at the Fermilab Tevatron seemed to be significantly larger than QCD calculations [1-10]. It took a while (see [11, 12] for a review) before these discrepancies could be resolved, through improvements in the accuracy of both the experimental measurements [13-15] and the theoretical predictions. In particular, from the theoretical side, the introduction of the "Fixed Order + Next-to-Leading Log" (FONLL) framework [16-18] has shown that the discrepancies are largely reduced if a consistent use is made of the fragmentation function information coming from $e^{+} e^{-}$data. From the experimental side, the extension of the $b$ production measurements to very small transverse momenta [13] has 
demonstrated good agreement with the fixed order QCD calculation [19] in a region where theoretical uncertainties due to fragmentation effects have very little relevance.

These successful comparisons notwithstanding, independent checks at a larger centreof-mass energy and with different measurements are of great interest. Firstly, low- $p_{T}$ production at the LHC energies probes values of the momentum fraction $x$ smaller than at the Tevatron, and challenges QCD in a dynamical region where potentially large higher-order corrections need to be resummed. This is particularly true of production at large rapidity, which pushes $x$ of one of the two initial-state partons to values of $x \lesssim 10^{-4}$, and which can be studied by forward detectors like LHCb and the ALICE muon spectrometer. Secondly, the greater beam energy and the high luminosities of the LHC can push the kinematic reach to much larger transverse momenta, exposing another interesting dynamical regime, where the resummation of logarithms of big ratios (such as $p_{T}^{Q} / m_{Q}, E_{T}^{j e t} / m_{Q}, p_{T}^{Q} / p_{T}^{Q \bar{Q}}$, etc.) may become crucial.

In parallel with the most recent comparisons with Tevatron data mentioned above, new theoretical tools have become available. The successful matching of next-to-leading order QCD calculations with parton shower Monte Carlos (PSMCs) has led to the MC@NLO [20, 21] and the POWHEG [22, 23] implementations, with matching to HERWIG [24-26] and to PYTHIA [27], which allow one to obtain predictions for fully exclusive observables while guaranteeing that inclusive quantities retain full NLO accuracy. Predictions can now therefore be evaluated within different frameworks (see also [28, 29] for an independent analysis) and compared among themselves as well as with the data.

The purpose of this paper is threefold. Firstly, we compare theoretical predictions obtained within the FONLL, MC@NLO and POWHEG frameworks for realistic observables (heavy mesons/hadrons, leptons from heavy hadrons, $J / \psi$ from $b$-hadron decays). Secondly, we compare these predictions, calculated within the cuts employed by the experimental analyses, with the available data. Thirdly, we provide a detailed public record for predictions that have been transmitted to the experimental community over the past two years, and that have been used in the comparisons with data that are documented in several experimental papers.

\section{Description of the theoretical frameworks}

\section{$2.1 \quad$ FONLL}

In the FONLL framework $[16,17]$, one matches fixed next-to-leading order (NLO) QCD [3032 ] with all-order resummation to next-to-leading log (NLL) accuracy in the limit where the transverse momentum $\left(p_{T}\right)$ of a heavy quark is much larger than its mass $(m)$ [33]. It allows one to calculate predictions for one-particle inclusive distributions of a heavy quark (or heavy hadron), while the degrees of freedom of the other particles in the event are integrated over. ${ }^{1}$ The accuracy of the FONLL calculation can be denoted as being NLO+NLL, with the understanding that the logarithms resummed up to next-to-leading accuracy are of

\footnotetext{
${ }^{1}$ We note that this implies that this approach does not allow the study of correlations between the heavy quark and antiquark.
} 
the form $\alpha_{s}^{n} \log ^{n}\left(p_{T} / m\right)$ and $\alpha_{s}^{n} \log ^{n-1}\left(p_{T} / m\right)$. The perimeter of the meaning of the name "FONLL" is often stretched to denote also the determination, from $e^{+} e^{-}$data [34], of the non-perturbative fragmentation parameters necessary for a successful description of physical differential distributions.

FONLL has been used extensively to predict bottom $[18,19]$ and charm [35] production data at the Tevatron and at RHIC [36]. In all cases satisfactory agreement between theory and data was found. The framework and the parameters employed in those predictions have not been modified since (except for a more systematic determination of the nonperturbative fragmentation parameters performed in [34], which confirmed earlier results).

A prediction for a single inclusive distribution (typically transverse momentum $\left(p_{T}\right)$, rapidity $(y)$ or pseudorapidity $(\eta))$ of a particle $\ell$ is obtained within FONLL as a numerical convolution of a perturbative cross section $d \sigma_{Q}^{F O N L L}$ with a non-perturbative fragmentation function $D_{Q \rightarrow H_{Q}}^{N P}$ and possibly a decay function $g_{H_{Q} \rightarrow \ell}^{\text {weak }}$ describing, for instance, the hadron weak decay into a lepton:

$$
d \sigma_{\ell}^{F O N L L}=d \sigma_{Q}^{F O N L L} \otimes D_{Q \rightarrow H_{Q}}^{N P} \otimes g_{H_{Q} \rightarrow \ell}^{\text {weak }} .
$$

The integral of the fragmentation functions $D_{Q \rightarrow H_{Q}}^{N P}$, for a given heavy-flavoured hadron $H_{Q}$, will be called the fragmentation fraction, $f\left(Q \rightarrow H_{Q}\right)$. The parameters for $D_{Q \rightarrow H_{Q}}^{N P}$ are best determined from $e^{+} e^{-}$collisions data, and a systematic survey is given in [34]. Weak decay spectra $g_{H_{Q} \rightarrow \ell}^{\text {weak }}$ and the corresponding branching ratios are similarly extracted from experimental data.

All the FONLL predictions published in this paper, as well as others corresponding to different cuts, input parameters or distributions, can be obtained from a publicly accessible web page [37].

\subsubsection{Non-perturbative fragmentation}

For completeness, we summarise here the parameters that we have used in this paper.

For bottom production, the functional form chosen for the parametrization of the non perturbative fragmentation function is a Kartvelishvili et al. distribution [38]:

$$
D_{b \rightarrow H_{b}}^{N P}=(\alpha+1)(\alpha+2) x^{\alpha}(1-x) .
$$

We choose $m_{b}=4.75 \mathrm{GeV}$ as central value for the bottom quark pole mass, and the range $m_{b}=4.5-5 \mathrm{GeV}$ for estimating the associated uncertainty. In this work we adopt the values of the fragmentation parameter $\alpha$ obtained in ref. [34], namely $\alpha=24.2$ for $m_{b}=4.75 \mathrm{GeV}$, $\alpha=26.7$ for $m_{b}=4.5 \mathrm{GeV}$ and $\alpha=22.2$ for $m_{b}=5 \mathrm{GeV} .{ }^{2}$ These parameters were fitted

\footnotetext{
${ }^{2}$ These parameters differ from those employed in [18, 19, 36], where $\alpha=29.1$ was used as a central value with $m_{b}=4.75 \mathrm{GeV}$, together with $\alpha=34$ with $m_{b}=4.5 \mathrm{GeV}$ and $\alpha=25.6$ with $m_{b}=5 \mathrm{GeV}$ (note that in the text of refs. $[19,36]$ the values for $\alpha$ corresponding to $m_{b}=4.5$ and $m_{b}=5 \mathrm{GeV}$ are unfortunately inverted, although the numerical results were obtained with the correct ones). The choice made in $[18,19,36]$ corresponded to using a fit to the $N=2$ Mellin moment of the $e^{+} e^{-}$data, whereas the values used in this paper correspond to a fit to the $N=5$ moment. The reason for a different choice is that the $N=5$ moment is more appropriate for describing steeply falling $p_{T}$ distributions like the ones found at large $p_{T}$ at the LHC. One should note, however, that switching from the old parameters set to the new one can be seen to lower the predictions by $5-10 \%$ at most: it does not affect therefore the good agreement with the data found in the past.
} 
in [34] to the LEP data relevant to the production of a mixture of $b$-hadrons [39, 40]. No data are available for the individual hadrons, for instance $B^{ \pm}$or $B^{0}$. One is therefore forced to assume a similar non-perturbative fragmentation for all of them. This assumption is likely fulfilled to a large extent, with the possible partial exception of $\Lambda_{b}$ production, which however only contributes a minor fraction of the $b$-hadron yield. We shall collectively denote the $b$-hadrons by $H_{b}$, and assume that each $b$ quark eventually produces a $b$-hadron, i.e. the fragmentation fraction $f\left(b \rightarrow H_{b}\right)$ is equal to one (and equivalently for the $\bar{b}$ ). The function in eq. (2.2) is normalised to one: when used for a specific state the appropriate fragmentation fraction (e.g. $\left.f\left(b \rightarrow B^{+}\right)\right)$must additionally be provided.

For charm production the situation is more complex. On one hand, experimental data are available for individual mesons $\left(D^{*}, D^{ \pm}, D^{0}\right.$ and $\left.\bar{D}^{0}, D_{s}\right)$. On the other, at least partial theoretical understanding exists on the differences and similarities in the fragmentation of a heavy quark into a pseudoscalar $(D)$ or a vector $\left(D^{*}\right)$ mesons (see e.g. [41]). This understanding can therefore be exploited in order to minimize the number of parameters to be extracted from experimental data. To this aim, in ref. [35] the non-perturbative fragmentation functions into different charmed mesons were constructed exclusively in terms of the $c \rightarrow D^{*}$ fragmentation, whose single parameter was extracted from ALEPH $e^{+} e^{-}$ data [42]. The decays of $D^{*}$ into $D$ states was modeled theoretically, and the various branching ratios were extracted from data. Primary $D$ production from $c$ fragmentation was described in terms of the same non-perturbative parameter fitted to $c \rightarrow D^{*}$ data, though a different functional form, as computed in [41], was used. The detailed results for the non-perturbative fragmentation functions $D_{c \rightarrow D^{*}}^{N P}, D_{c \rightarrow D^{+}}^{N P}$ and $D_{c \rightarrow D^{0}}^{N P}$ are given in eqs. (10), (9) and (5) of [35] respectively, and we do not repeat them here for brevity. They depend (besides the branching ratios of experimental origin) on a single non-perturbative parameter $r$ determined from data. We used the values $r=0.1$ with $m_{c}=1.5 \mathrm{GeV}, r=0.06$ with $m_{c}=1.3 \mathrm{GeV}$ and $r=0.135$ with $m_{c}=1.7 \mathrm{GeV}$. These values also correspond to a $N=5$ fit as detailed in [34].

\subsubsection{Theoretical uncertainties}

The 'central' FONLL prediction is computed by setting the renormalisation and factorisation scales equal to the transverse mass, $\mu_{R, F}=\mu_{0} \equiv \sqrt{p_{T}^{2}+m^{2}}$ : here, $m$ and $p_{T}$ are the heavy quark mass and transverse momentum respectively. The theoretical uncertainty is estimated as a combination of factorisation and renormalisation scale variations, heavy quark mass variation, and uncertainty associated with Parton Distribution Functions (PDFs). The three uncertainties will normally be combined in quadrature.

- In order to avoid accidental compensation between the $\mu_{F}$ and the $\mu_{R}$ dependence of the cross section, which may occur if the two scales are set equal, we compute the scale uncertainty by varying them independently over the range $0.5 \leq \xi_{R, F} \leq 2$, with the constraint $0.5 \leq \xi_{R} / \xi_{F} \leq 2$, where $\xi_{R, F} \equiv \mu_{R, F} / \mu_{0}$. In practice, the cross section is evaluated (using the central mass value and PDF set) in the seven points

$$
\left(\xi_{R}, \xi_{F}\right) \in\{(1,1),(0.5,0.5),(2,2),(0.5,1),(1,0.5),(2,1),(1,2)\}
$$


and the envelope is taken. This envelope defines, at each point in the distribution one is considering, the two extremes of

$$
d \sigma_{-\Delta_{-, \text {scales }}^{+\Delta}}^{+ \text {scales }}
$$

- The three mass values $m=1.5,1.3$ and $1.7 \mathrm{GeV}$ are used for charm, and $m=4.75$, 4.5 and $5 \mathrm{GeV}$ for bottom. Non-perturbative parameters are adjusted for each mass to their appropriate value, ${ }^{3}$ and the cross section is evaluated setting $\xi_{R, F}=1$ and with the central PDF set. The envelope is then taken, defining

$$
d \sigma_{-\Delta_{-, \text {mass }}}^{+\Delta_{+, \text {mass }}} \text {. }
$$

- The PDF uncertainty, where available, is evaluated (setting $\xi_{R, F}=1$, and the mass equal to the central value) as suggested by the specific PDF set used (see e.g. [43]), yielding

$$
d \sigma_{-\Delta_{-, P D F}^{+}}^{+\Delta_{+, P D F}} .
$$

Our default PDF set will be CTEQ6.6 [44], unless otherwise stated.

The full uncertainty band of the FONLL predictions will be given by

$$
d \sigma_{-\Delta_{-}^{+}}^{+\Delta_{+}}
$$

with

$$
\Delta_{ \pm}=\sqrt{\Delta_{ \pm, \text {scales }}^{2}+\Delta_{ \pm, \text {mass }}^{2}+\Delta_{ \pm, P D F}^{2}}
$$

\section{$2.2 \quad \mathrm{NLO}+\mathrm{PS}$ approaches}

Heavy flavour production has been available for quite some time in NLO+PS (Next-toleading order plus parton shower) models, namely in MC@NLO [21] and in POWHEG [23]. These programs, in conjunction with existing parton shower programs, are capable of generating fully exclusive final states, maintaining the next-to-leading order accuracy for inclusive observables.

We remark that, unlike the FONLL approach, these generators resum only a subset of all contributions enhanced by large logarithms of the ratio of the transverse momentum of the heavy quark over its mass. In particular, the so called gluon splitting and flavour excitation production mechanisms are implemented only at order $\mathcal{O}\left(\alpha_{S}^{3}\right)$, i.e. the lowest order contributions to these processes. In contrast, FONLL includes for these contributions all terms of order $\alpha_{S}^{2} \times\left(\alpha \log p_{T} / m\right)^{n}$ and $\alpha_{S}^{3} \times\left(\alpha_{S} \log p_{T} / m\right)^{n}$. On the other hand, at small and moderate transverse momenta, the NLO+PS approaches are superior to FONLL, since they have the same accuracy in this region but, unlike FONLL, provide a complete and fully exclusive description of the final state.

\footnotetext{
${ }^{3}$ Note that this adjustment translates into a non negligible reduction of the sensitivity of the hadron-level cross section on the heavy quark mass value at large transverse momentum, as one may expect from the fact that neither the heavy quark mass nor the non-perturbative fragmentation are physical observables, and therefore their variations must compensate each other in their interplay.
} 
In the present comparison, the NLO+PS methods should be viewed as approaches that work at small and moderate energies, and that are bound to fail at some large transverse momentum scale. To some extent, the present work helps to assess the range of validity of these approaches.

It should be also kept in mind that the non-perturbative part of the shower that leads to the formation of the $b$ hadrons is handled by the Parton Shower program alone. The corresponding parameters are tuned using final-state observables reconstructed with particles emerging from the parton shower, but with the hard production cross section of the shower Monte Carlo, i.e. not that of the next-to-leading order ones used in the NLO+PS methods. This is in contrast with what is done in FONLL, where use is made of a non-perturbative fragmentation function which is fitted to $e^{+} e^{-}$data with a theoretical calculation based on the very same underlying FONLL approach.

We point out that the parameters that control cluster decays in Fortran HERWIG [26] (PSPLT, CLDIR, and CLSMR) may be assigned (depending on the tuning adopted) specific values for $b$-flavoured clusters. On the other hand, $c$-flavoured cluster decays are treated in the same manner as light quark ones. Although one can envisage to relax this constraint, and to introduce and tune parameters relevant to $c$-flavoured clusters only, such a possibility is not given in the official HERWIG versions, and has not been considered here.

\subsubsection{MC@NLO}

The MC@NLO formalism has been introduced in ref. [20], and aims at a consistent matching between NLO QCD corrections for a given process, and parton showers. The relevant technical details are given in the quoted references, and we shall omit them here. We limit ourselves to recall that in the context of MC@NLO the matching prescription amounts to modifying the short-distance cross sections relevant to the NLO computation, by including the so-called Monte Carlo (MC) subtraction terms, that are responsible for removing any double counting at the NLO. The MC subtraction terms can be computed in a processindependent manner, but they are still dependent on the particular PSMC one adopts for the shower phase. In other words, each PSMC requires a set of MC subtraction terms, which can be obtained by formally expanding the PSMC results to the same order in $\alpha_{\mathrm{S}}$ as the corresponding NLO contribution to the parton-level cross section (i.e. that of the real-emission matrix elements). Furthermore, their structures are such that all non trivial process-specific information is contained in the Born matrix elements. These matrix elements are multiplied by kernels whose analytic forms depend solely on the shower variables used by the PSMC to generate the elementary branchings, and on the identities of the partons involved in such branchings.

Although ref. [20] formulated the solution of NLO+PS matching in general terms, practical applications there and in subsequent papers have been restricted to the choice of Fortran HERWIG [24-26] as PSMC. Recently, MC subtraction terms have been computed which are relevant to the matching with Herwig $++[45]$ and, for processes that feature only initial-state emissions, with PYTHIA 6.4 [27] (see refs. [46] and [47] respectively ${ }^{4}$ ). In

\footnotetext{
${ }^{4}$ The matching with PYTHIA (including the $p_{\mathrm{T}}$-ordered shower versions) for a generic process is being achieved in the context of aMC@NLO — see http://amcatnlo.cern.ch for more information on this project.
} 
the present work, the MC@NLO results have been obtained with Fortran HERWIG (v6.520, which, for the processes considered here, gives results identical to those of v6.510), using the implementation of $Q \bar{Q}$ production presented in ref. [21]. We point out that, although such an implementation technically only requires the mass of the heavy quark to be larger than $\Lambda_{\mathrm{QCD}}$, the conservative choice has been made of not including $c \bar{c}$ production in the public MC@NLO package. This is due to the fact that, because of the relative lightness of the charm quark, contributions may be important where a light-parton scattering such as $g g \rightarrow g g$ initiates showers that eventually feature a $g \rightarrow c \bar{c}$ branching. These kind of contributions are not included in an NLO+PS matched computation, unless the aforementioned branching is the first occurring in a given shower. Although this mechanism is relevant also in the case of $b \bar{b}$ production, it is expected to be less important there than in the case of $c \bar{c}$ final states. It is therefore interesting to compare NLO+PS predictions with FONLL, which does take correctly into account the contributions discussed above. The default scale choice for $Q \bar{Q}$ production in MC@NLO is

$$
\mu_{0}^{2}=\frac{1}{2}\left(m_{\mathrm{T}}^{2}(Q)+m_{\mathrm{T}}^{2}(\bar{Q})\right), \quad m_{\mathrm{T}}^{2}=p_{\mathrm{T}}^{2}+m^{2},
$$

where $p_{\mathrm{T}}$ is the transverse momentum w.r.t. the beam line (note that, in the real-emission contributions to the NLO cross section, the transverse momentum of the $Q$ is generally not equal to that of the $\bar{Q}$ ).

\subsubsection{POWHEG}

The POWHEG implementation of heavy flavour production is described in detail in ref. [23]. It is now available at the POWHEG BOX website, http://powhegbox.mib.infn.it/. It can be used to generate events with either $t \bar{t}, b \bar{b}$ or $c \bar{c}$ pairs, and it is based upon the heavy flavour production next-to-leading order calculation of refs. [30-32, 48]. The output is an event file (in the Les Houches format [49]), that can be fed through any Shower Monte Carlo program that complies with the requirements of the Les Houches Interface for User Processes (LHIUP), like the Fortran and $\mathrm{C}++$ versions of PYTHIA and HERWIG, in order to generate complete events.

The default scale choice used in this implementation is given by $\sqrt{p_{T}^{2}+m^{2}}$, where $p_{T}$ is the transverse momentum of the heavy flavour in the underlying Born configuration. This is the structure of the $Q \bar{Q}$ event before the hardest radiation has been generated, as defined in refs. $[50,51]$. It is obtained as follows. One performs a longitudinal boost of the heavy quark pair system such that the system has zero rapidity after the boost, then a transverse boost is performed such that the transverse momentum of the system vanishes, and then the inverse of the initial longitudinal boost is performed. Thus, although the total cross section in POWHEG corresponds to the NLO result, as in MC@NLO, one cannot expect the two generators to yield identical cross sections because of the different scale choice.

POWHEG has been interfaced to PYTHIA version 6.4.25, and HERWIG 6.510, keeping always the default values of the parameters in the Monte Carlo. A non negligible sensitivity to the Monte Carlo tune is expected for the observables considered in this work. An example is given in figure 1 , where $D^{+}$and $B^{+}$production as predicted by POWHEG with PYTHIA 

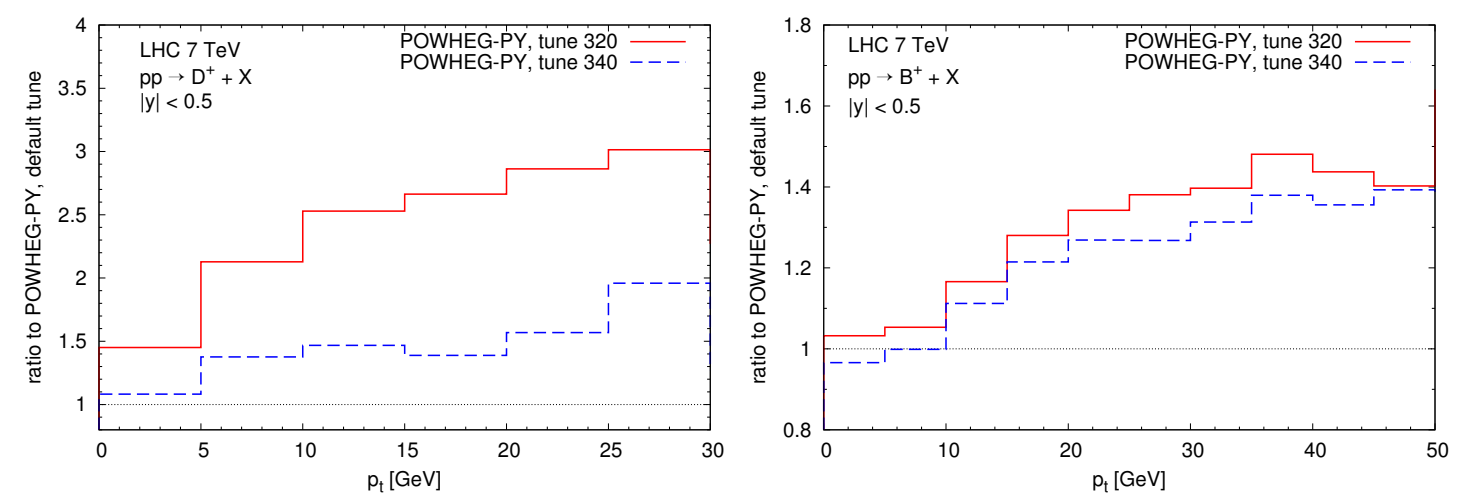

Figure 1. Sensitivity of POWHEG with PYTHIA predictions to the tune used in PYTHIA for $D^{+}$ (left plot) and $B^{+}$(right plot) transverse momentum distributions in the central rapidity region. At large rapidities, we observe effects of similar size or larger.

is studied for different PYTHIA tunes in $|y|<0.5$. At large rapidities the sensitivity to the PYTHIA tune is similar, if not larger. A complete study of the tune dependence is outside the scope of the present paper, and all Monte Carlo results presented in this paper will employ the default tune, unless explicitly stated.

\section{Numerical predictions and comparisons}

\subsection{Open charm production}

We begin by considering the production of $D$ mesons. The FONLL framework and its nonperturbative parameters are described in section 2.1.1. In the following we concentrate on the production of $D^{+}$mesons (the others being largely similar) and we use a value of the fragmentation fraction of $f\left(c \rightarrow D^{+}\right)=0.238$ with FONLL. The left plot of figure 2 shows the central FONLL prediction, compared with MC@NLO, POWHEG with PYTHIA (denoted by POWHEG-PY) and POWHEG with HERWIG (denoted by POWHEG-HW). The right plot shows the scale and PDF contributions to the FONLL uncertainty, as well as the overall systematics. The ALICE data from ref. [52] are also compared to the theoretical prediction, showing a good agreement within the large systematics. We note that all four theoretical predictions are in fair agreement with each other within the overall uncertainty. The large dependence, in shape as well as overall normalisation, of the Monte Carlo results on the specific tune, as shown in section 2.2.2, should also be kept in mind. Differences appear, on the other hand, in the forward region $4.5<|y|<5$, as shown in figure 3. POWHEG-PY agrees well with FONLL, while the shower evolution carried out with HERWIG (whether in the MC@NLO or in the POWHEG framework) leads to a harder spectrum. These differences largely exceed the systematics quoted from the FONLL calculation, in particular if we consider that the sightly bigger uncertainty at large $p_{T}$ is mostly due to the PDFs, and is therefore entirely correlated among the different predictions. It will be very interesting to see the first data on forward $D^{+}$production at large $p_{T}$ from $\mathrm{LHCb}$. 

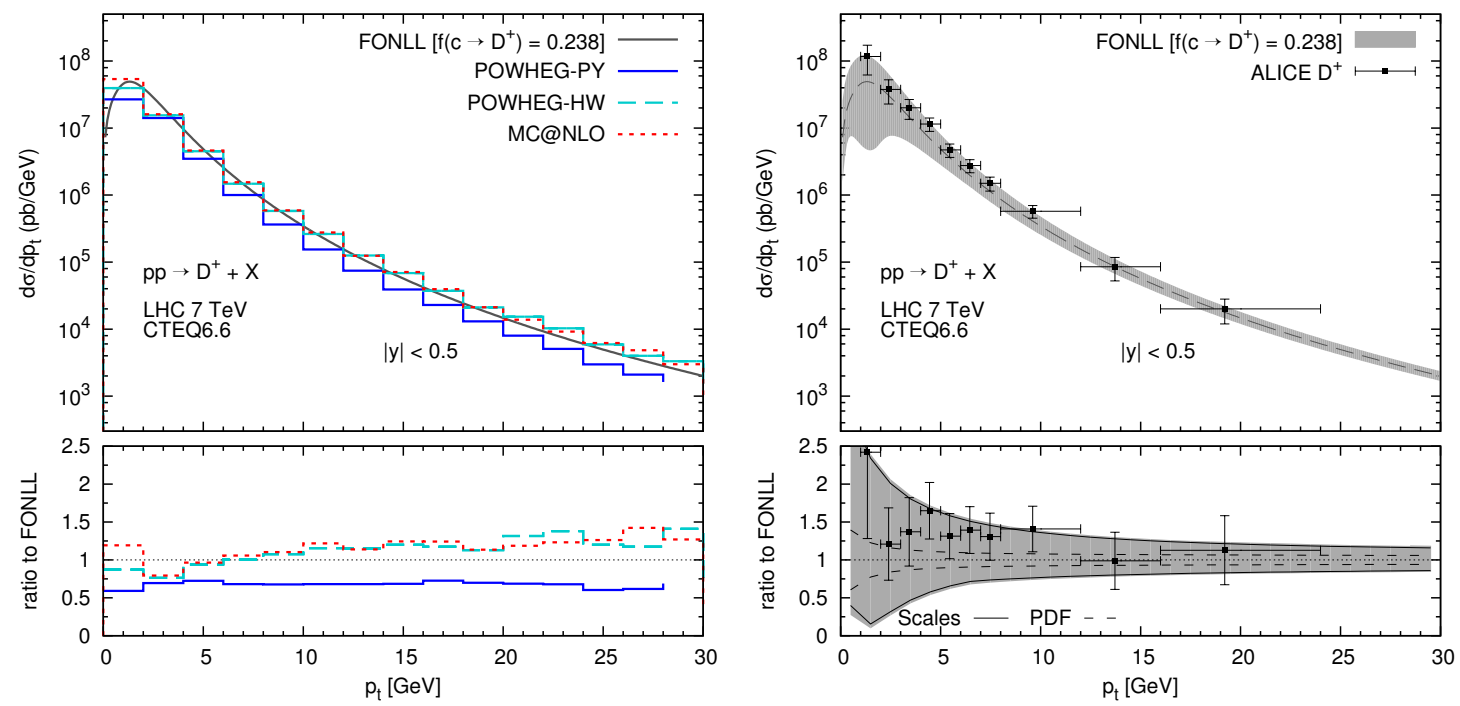

Figure 2. Transverse momentum distribution of $D^{+}$mesons at central rapidity, $|y|<0.5$. Left plot: comparison among the central predictions of our four benchmark calculations, FONLL, MC@NLO, and POWHEG with PYTHIA or HERWIG showers. Right plot: theoretical systematics for the FONLL calculation, and the comparison with data from ALICE [52]. For the systematics we show the individual scale and PDF components, as well as the combined total (which includes mass variation, as described in the text).
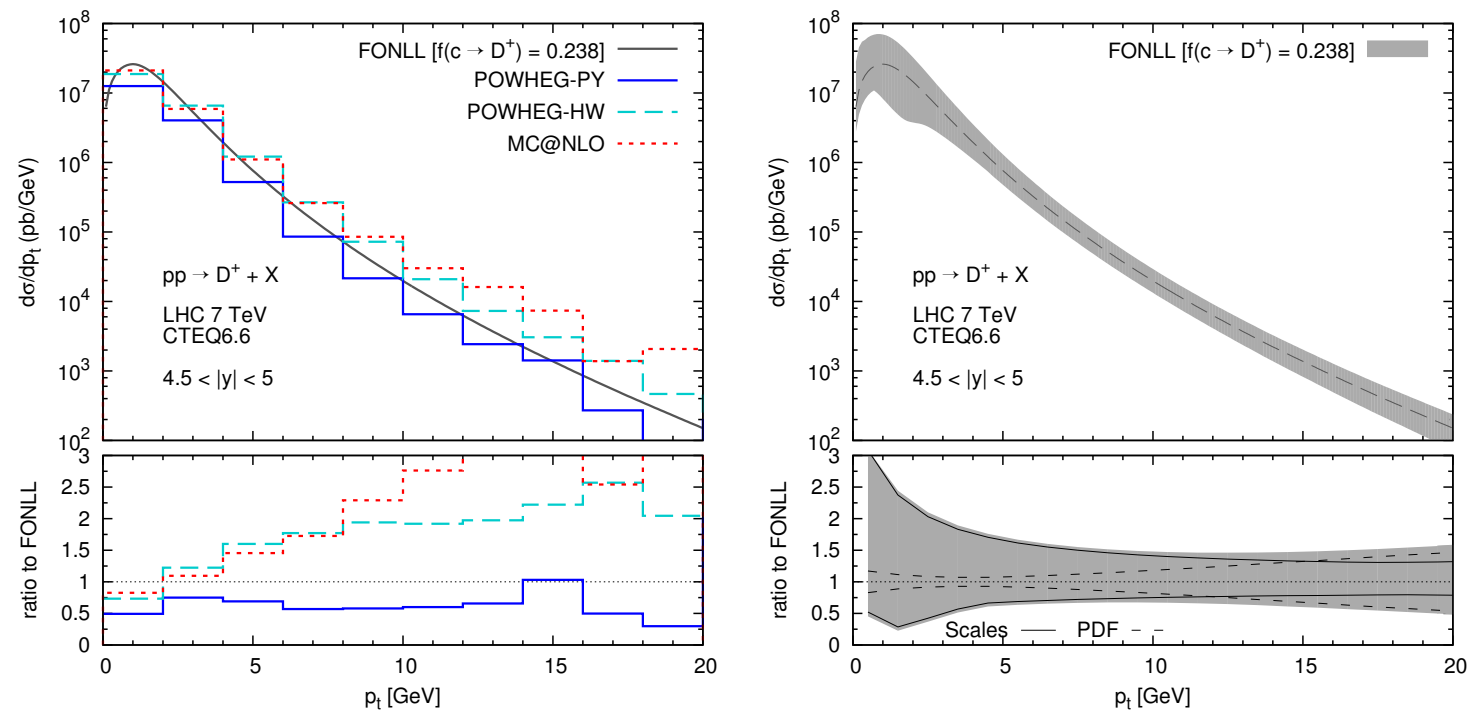

Figure 3. Same theoretical distributions as in figure 2, for the forward rapidity region $4.5<|y|<5$.

Additional data on $D^{0}$ and $D^{*+}$ production in the central region, and comparisons with the FONLL predictions, are reported by the ALICE Collaboration in figure 5 of [52]. Similar data, at $\sqrt{S}=2.76 \mathrm{TeV}$, are reported in [53]. Preliminary data from ATLAS are also available, and compared to FONLL in figure 1 and 2 of [54]. In either case a fair agreement is found, with the data mostly centred on the upper edge of the theoretical 

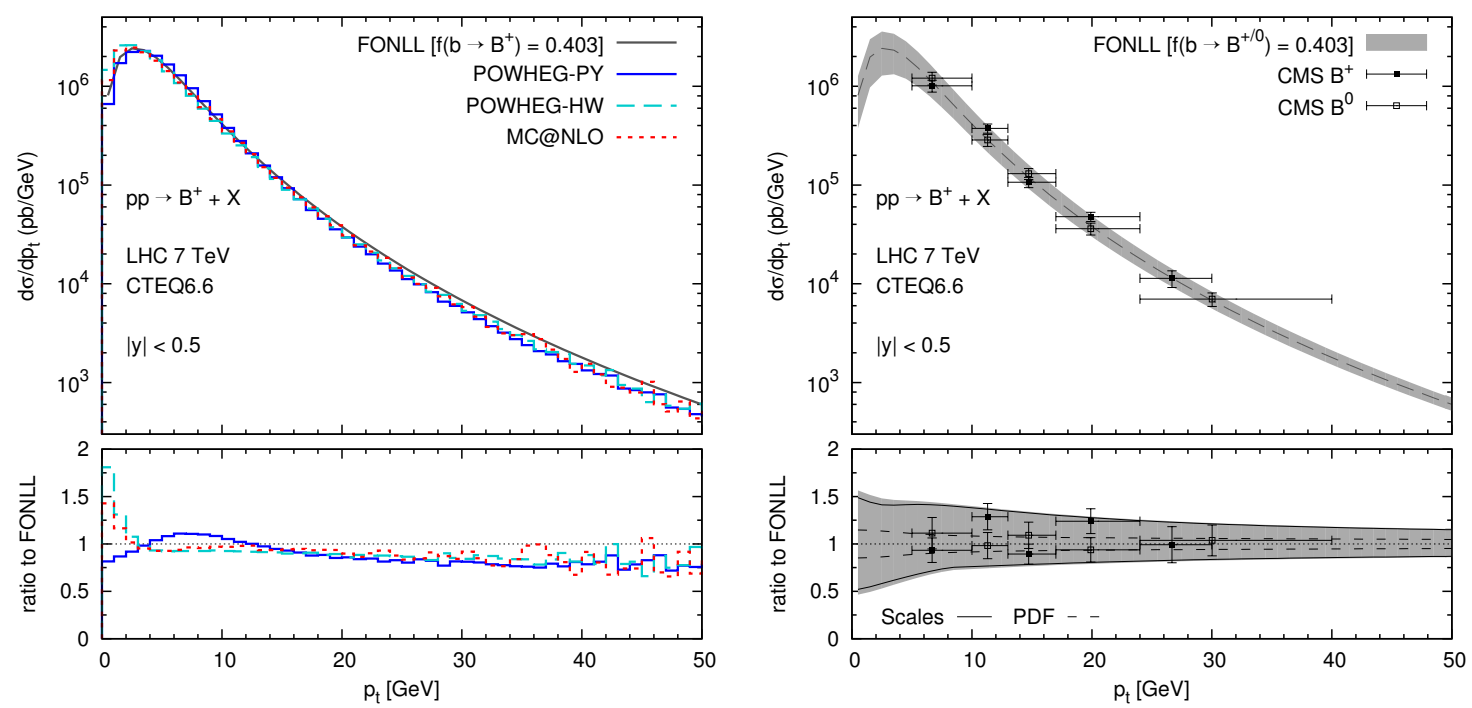

Figure 4. Transverse momentum distribution of $B^{+}$mesons at central rapidity, $|y|<0.5$. Left plot: comparison among the central predictions of our four benchmark calculations, FONLL, MC@NLO, and POWHEG with PYTHIA or HERWIG showers. Right plot: theoretical systematics for the FONLL calculation, and the comparison with data from CMS [55, 56], rescaled to the $\left|y^{B}\right|<0.5$ region. For the systematics we show the individual scale and PDF components, as well as the combined total.

uncertainty band, mirroring quite closely the comparisons with the Tevatron $D$ mesons data [35]. This may indicate a preference of the data for a value of the charm mass smaller than our default of $1.5 \mathrm{GeV}$.

\subsection{Open bottom production from inclusive and fully-reconstructed $H_{b} \rightarrow$ $D+X$ decays}

The theoretical predictions for central production of $B^{+}$mesons $(|y|<0.5)$ are presented in figure 4 . The quality of the agreement among the various predictions is similar to the one seen in the $D^{+}$case above. Other features, like - at large rapidity - the progressively larger theoretical uncertainty as a consequence of a larger PDF uncertainty, or the larger variance between the NLO+PS and the FONLL predictions (see figure 5), are also similar. The potential sensitivity of the Monte Carlo results to the specific tune used (though smaller than in the $D$-mesons case, see figure 1), should be kept in mind in this case too.

The first measurement of $b$-hadron production at the $\mathrm{LHC}$ was performed by the $\mathrm{LHCb}$ Collaboration [57]. The pseudorapidity distribution in the region $2 \leq \eta \leq 6$ was shown to be in good agreement with NLO and FONLL predictions (see figure 5 of [57]). The measured total cross section in this region was found to be (averaging over $b$ and $\bar{b}$ hadrons, and using the values of $b$-hadron fractions measured at the Tevatron $)^{5}$

$$
\sigma^{\mathrm{LHCb}}\left(p p \rightarrow H_{b}, 2 \leq \eta \leq 6\right)=89.6 \pm 6.4 \pm 15.5 \mu \mathrm{b}
$$

\footnotetext{
${ }^{5}$ Note that, as explained in [57], the measurement changes to $75.3 \pm 5.4 \pm 10.0 \mu b$ if, instead, $b$-hadron fractions measured at LEP are used in converting the number of events to an $H_{b}$ cross section.
} 

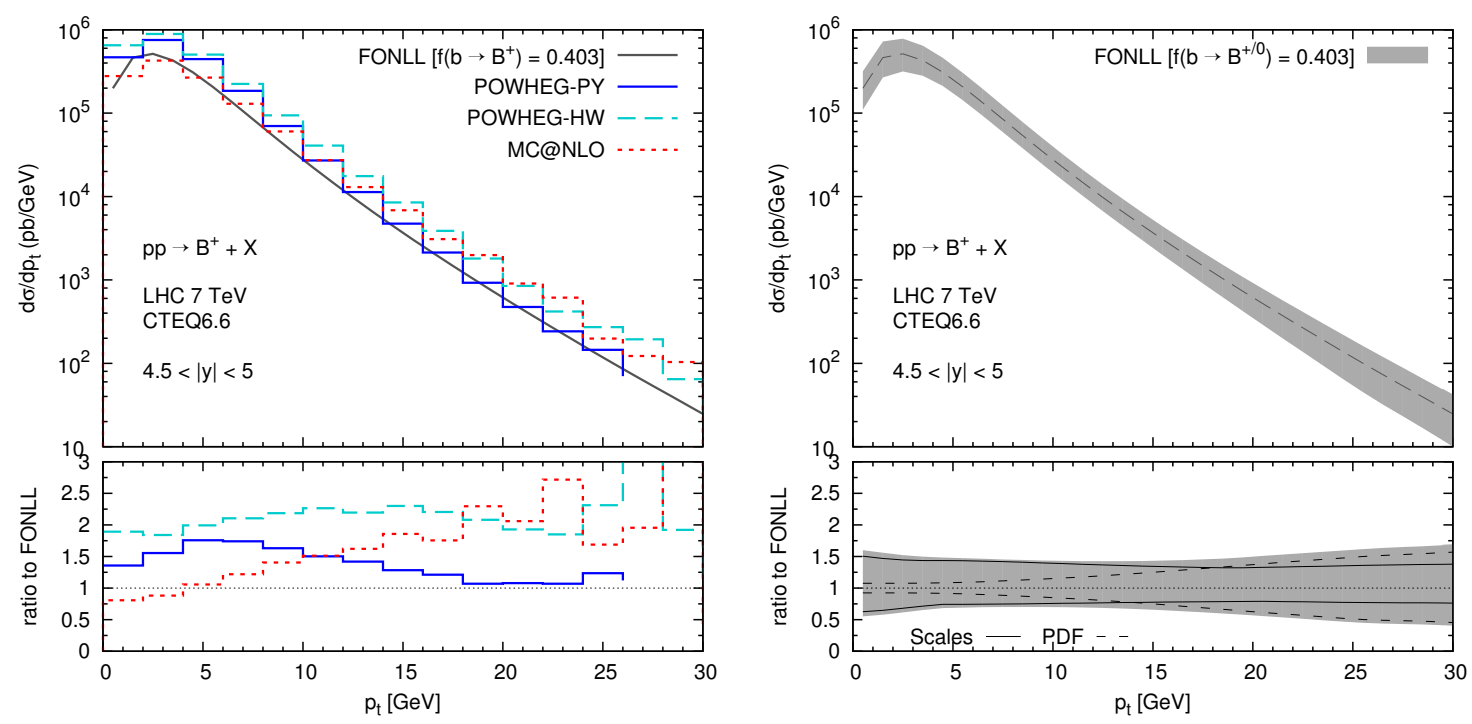

Figure 5. Same theoretical distributions as in figure 4, for the forward rapidity region $4.5<|y|<5$.

to be compared with the FONLL prediction ${ }^{6}$

$$
\sigma^{\mathrm{FONLL}}\left(p p \rightarrow H_{b}, 2 \leq \eta \leq 6\right)=70.8_{-24.4}^{+33.3} \mu b .
$$

As remarked above, the contribution of $b$-baryons are included in this theoretical value assuming they fragment like the $B$ mesons.

More recently, LHCb has published the measurement of the $B^{ \pm}$production cross section in the $2<y<4.5$ rapidity region [58], using fully reconstructed $B^{ \pm} \rightarrow J / \psi K^{ \pm}$decays:

$$
\sigma^{\mathrm{LHCb}}\left(p p \rightarrow B^{ \pm}, 0<p_{T}<40 \mathrm{GeV}, 2<y<4.5\right)=41.4 \pm 1.5 \pm 3.1 \mu b
$$

to be compared with the FONLL prediction (which includes a fragmentation fraction $f(b \rightarrow$ $\left.\left.B^{-}\right)=0.403\right)$

$$
\sigma^{\mathrm{FONLL}}\left(p p \rightarrow B^{ \pm}, 0<p_{T}<40 \mathrm{GeV}, 2 \leq y \leq 4.5\right)=40.1_{-14.5}^{+19.0} \mu b .
$$

Good agreement in seen also in the differential $p_{T}^{B^{ \pm}}$spectra, in the range up to $40 \mathrm{GeV}$, as shown in figure 2 of [58].

The CMS collaboration has published measurements of transverse momentum and rapidity distributions of $B^{+}[55], B^{0}[56]$ and $B_{s}^{0}[59]$ mesons. They report, for the total visible cross sections at $7 \mathrm{TeV}$ LHC,

$$
\begin{aligned}
& \sigma^{\mathrm{CMS}}\left(p p \rightarrow B^{0}, p_{T}^{B}>5 \mathrm{GeV},\left|y^{B}\right|<2.2\right)=33.2 \pm 2.5 \pm 3.5 \mu b \\
& \sigma^{\mathrm{CMS}}\left(p p \rightarrow B^{+}, p_{T}^{B}>5 \mathrm{GeV},\left|y^{B}\right|<2.4\right)=28.1 \pm 2.4 \pm 2.0 \pm 3.1 \mu b \\
& \sigma^{\mathrm{CMS}}\left(p p \rightarrow B_{s}^{0}, 8<p_{T}^{B}<50 \mathrm{GeV},\left|y^{B}\right|<2.4\right) \times \mathrm{BR}\left(B_{s}^{0} \rightarrow J / \psi \phi\right)=6.9 \pm 0.6 \pm 0.6 \mathrm{nb},
\end{aligned}
$$

\footnotetext{
${ }^{6}$ Here and elsewhere, where the quoted FONLL predictions already appeared in experimental papers. They were originally calculated according to the framework defined in this paper, and provided as private communications to the experimental collaborations.
} 
to be compared with the FONLL predictions

$$
\begin{aligned}
& \sigma^{\mathrm{FONLL}}\left(p p \rightarrow B^{0}, p_{T}^{B}>5 \mathrm{GeV},\left|y^{B}\right|<2.2\right)=25.5_{-7.1}^{+10.5} \mu b \\
& \sigma^{\mathrm{FONLL}}\left(p p \rightarrow B^{+}, p_{T}^{B}>5 \mathrm{GeV},\left|y^{B}\right|<2.4\right)=27.2_{-7.5}^{+11.2} \mu b \\
& \sigma^{\mathrm{FONLL}}\left(p p \rightarrow B_{s}^{0}, 8<p_{T}^{B}<50 \mathrm{GeV},\left|y^{B}\right|<2.4\right) \times \mathrm{BR}\left(B_{s}^{0} \rightarrow J / \psi \phi\right)=4.5_{-1.1}^{+1.7} \pm 1.6 n b .
\end{aligned}
$$

The FONLL uncertainties in eqs. (3.8)-(3.10) are due to renormalisation and factorisation scales, heavy quark masses and PDF, as detailed in section 2.1.2. Of these three sources, the first is largely dominant. In the FONLL predictions the fragmentation fractions $f(b \rightarrow$ $\left.B^{0}\right)=f\left(b \rightarrow B^{-}\right)=0.403$ and $f\left(b \rightarrow B_{s}^{0}\right)=0.11[60]$ have been included. These values, leading to a ratio $f_{s} / f_{d}=0.273$, are consistent with the recent direct measurement of this ratio by LHCb [61]: $f_{s} / f_{d}=0.267_{-0.020}^{+0.021}$. Additionally, $\operatorname{BR}\left(B_{s}^{0} \rightarrow J / \psi \phi\right)=(1.4 \pm 0.5) \times$ $10^{-3}[60]$ has been used, and the second uncertainty in the FONLL prediction of eq. (3.10) for $B_{s}^{0}$ production reflects the large uncertainty of this measured branching ratio.

A good agreement with the experimental measurements within the respective uncertainties can be observed, confirming the latest comparisons between theory and Tevatron data which showed no significant excess in bottom hadroproduction compared to theoretical predictions. The $p_{T}$ spectra measured by CMS are compared to various predictions in figure 2 of ref. [56] and figure 2 of ref. [55]. The comparison of these data, rescaled to the rapidity region $|y|<0.5$, with the FONLL predictions is shown in the right panel of figure 4 .

\subsection{Open bottom production from inclusive $H_{b} \rightarrow J / \psi$ and $H_{b} \rightarrow \psi(2 S)$ decays}

The predictions for non-prompt $J / \psi$ and $\psi(2 S)$, i.e. coming from $b$-hadron decays, is obtained in FONLL by convoluting the distribution for $b$-hadron production with a phenomenological spectrum, obtained from experimental data [62] (that describes the momentum distribution of the $J / \psi$ and the $\psi(2 S)$ in $B$-meson decays), and by multiplying it by the appropriate branching fraction (again obtained from experimental measurements). In using decay spectra from $B$ meson decays and applying them to all $b$-hadron decays we make the reasonable assumption that a potential difference in the small fraction $(\sim 10-20 \%)$ of non-meson $b$-hadrons is going to be inconsequential. Moreover, it should be noted that strictly speaking ref. [62] gives the momentum of the quarkonium in the $\Upsilon(4 S)$ rest frame rather than in the $B$-meson one. The difference has a small rms spread $(0.12 \mathrm{GeV}$ according to [62]) and is not expected to affect significantly the final quarkonium spectrum in our convolution. In the following we shall use the branching ratio $\operatorname{BR}(b \rightarrow h)$, defined as $\mathrm{BR}(b \rightarrow h)=\sum_{H_{b}} f\left(b \rightarrow H_{b}\right) \times \mathrm{BR}\left(H_{b} \rightarrow h+X\right)$, summed over all relevant b-hadrons $H_{b}$.

The fit to the $B \rightarrow J / \psi$ decay spectrum from figure 6 of [62] had been performed in preparation of ref. [19], where non-prompt $J / \psi$ distribution in $p \bar{p}$ collisions at the Fermilab Tevatron had been calculated, and found in good agreement with the data, as well as with the MC@NLO predictions (see figure 5 of [19]). This paper uses the same spectrum. The 

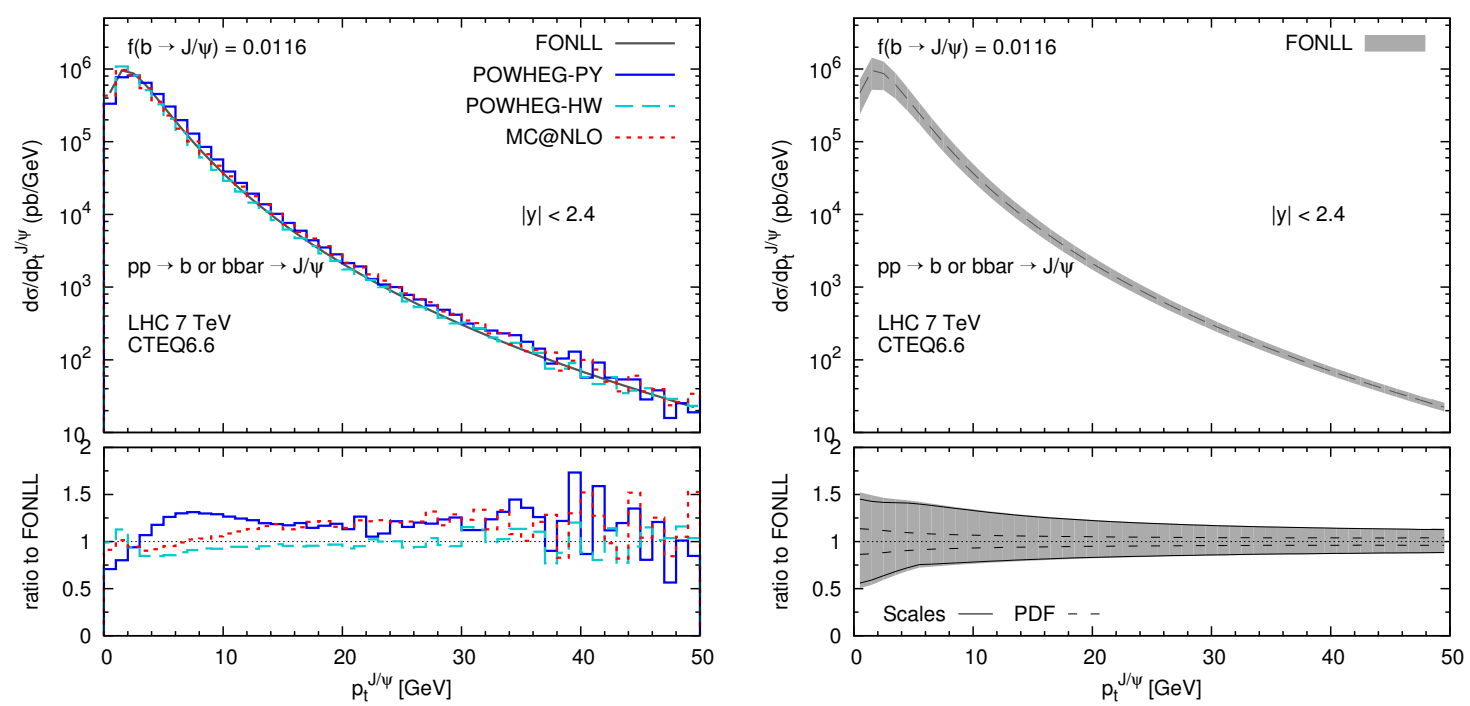

Figure 6. Transverse momentum distribution of non-prompt $J / \psi$ in the central rapidity region $|y|<2.4$. Left plot: comparison among the central predictions of our four benchmark calculations, FONLL, MC@NLO, POWHEG-HW and POWHEG-PY. Right plot: theoretical systematics for the FONLL calculation.

fit to the $\psi(2 S)$ spectrum from figure 8 of [62] has instead been performed for this paper, and predictions for the non-prompt $\psi(2 S)$ within FONLL are given here for the first time.

Figure 6 compares predictions for the transverse momentum distributions for the nonprompt $J / \psi$ and $\psi(2 S)$ production in the central rapidity region $|y|<2.4$. In this case the prediction from the POWHEG-PY implementation differ quite markedly from the others, and data could provide discriminating power.

The first experimental measurements at $7 \mathrm{TeV}$ from CMS [63, 64], LHCb [65] and ATLAS [66] have been compared to FONLL predictions and generally found in fairly good agreement. For example, LHCb [65], after separating the prompt and $b$-decay contributions, measures:

$$
\sigma^{\mathrm{LHCb}}\left(J / \psi \text { from } H_{b}, p_{T}<14 \mathrm{GeV}, 2<y<4.5\right)=1.14 \pm 0.01 \pm 0.16 \mu b
$$

to be compared with the FONLL prediction (which includes a branching fraction $\mathrm{BR}(b \rightarrow$ $J / \psi=0.0116)$

$$
\sigma^{\mathrm{FONLL}}\left(J / \psi \text { from } H_{b}, p_{T}<14 \mathrm{GeV}, 2<y<4.5\right)=1.16_{-0.42}^{+0.55} \mu b
$$

The FONLL predictions describe also very well the $J / \psi p_{T}$ spectrum, measured in the range $0-13 \mathrm{GeV}$, as shown in figure 9 of [65].

More recently, ALICE [67] reported:

$$
\sigma^{\mathrm{ALICE}}\left(J / \psi \text { from } H_{b}, p_{T}>1.3 \mathrm{GeV},|y|<0.9\right)=1.26 \pm 0.33{ }_{-0.28}^{+0.23} \mu b
$$

to be compared with the FONLL prediction

$$
\sigma^{\text {FONLL }}\left(J / \psi \text { from } H_{b}, p_{T}>1.3 \mathrm{GeV},|y|<0.9\right)=1.33{ }_{-0.48}^{+0.59} \mu b
$$




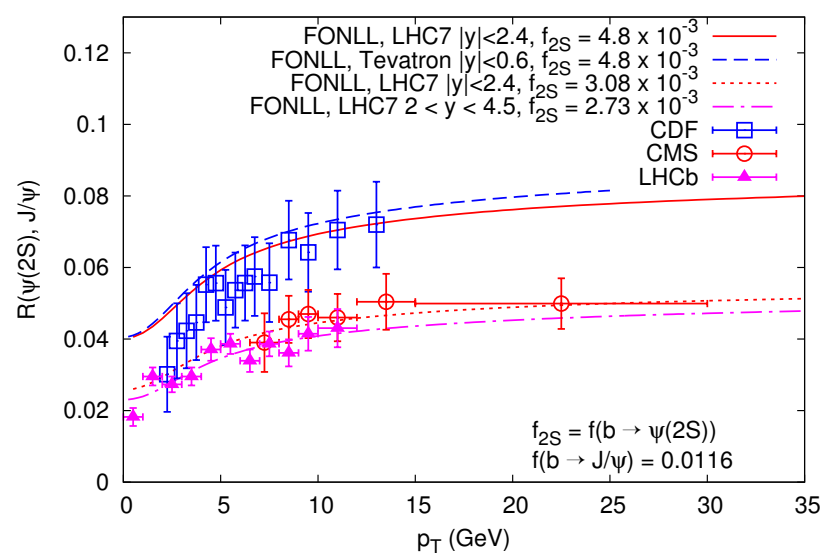

Figure 7. FONLL prediction of the ratio of the cross section for non-prompt (i.e. from $b$-hadrons) production of $\psi(2 S)$ and $J / \psi$ (each multiplied by its own branching ratio into muons) as a function of their transverse momentum, compared to experimental data from CDF [13] at the Tevatron and CMS [64] and LHCb [68] at the LHC.

A possible exception to the generally good agreement is the observation, made by CMS in [64], that the experimental cross section for non-prompt $J / \psi$ and $\psi(2 S)$ production tends to fall off at large transverse momentum slightly faster than the FONLL prediction. More data at even larger $p_{T}$ will help clarify this issue.

In the same paper, CMS note that the measured non-prompt $\psi(2 S)$ production is quite smaller than the FONLL prediction evaluated using the central value of the branching fraction $\operatorname{BR}(b \rightarrow \psi(2 S))=4.8 \times 10^{-3}[60]$. In order to better understand this potential discrepancy, it is useful to consider the ratio

$$
R(\psi(2 S), J / \psi) \equiv \frac{\frac{d \sigma}{d p_{T}}(b \rightarrow \psi(2 S)) \operatorname{BR}\left(\psi(2 S) \rightarrow \mu^{+} \mu^{-}\right)}{\frac{d \sigma}{d p_{T}}(b \rightarrow J / \psi) \operatorname{BR}\left(J / \psi \rightarrow \mu^{+} \mu^{-}\right)}
$$

as a function of the transverse momentum of the quarkonium. Figure 7 shows the FONLL predictions for this ratio, compared to data from CDF at the Tevatron [13] and CMS [64] and $\mathrm{LHCb}[68]$ at the LHC. One can see that the agreement with the Tevatron data is acceptable when one uses $\operatorname{BR}(b \rightarrow \psi(2 S))=(4.8 \pm 2.4) \times 10^{-3}[60]$ and considers its large $(\mathrm{O}(50 \%))$ uncertainty, which is not shown in the plot. The data from the CMS and the LHCb collaborations seem instead to be somewhat at variance with the CDF ones whereas, according to the FONLL prediction, no large difference should be present. One could still exploit the large uncertainty of $\mathrm{BR}(b \rightarrow \psi(2 S))$ to lower significantly the theoretical prediction and make it compatible with all measurements within the uncertainties. In fact, CMS have used their measurement of this ratio and the comparison to theoretical predictions to extract [64] a new value for the $\mathrm{BR}(b \rightarrow \psi(2 S))$ branching fraction,

$$
\mathrm{BR}(b \rightarrow \psi(2 S))^{\mathrm{CMS}}=(3.08 \pm 0.12 \pm 0.13 \pm 0.42) \times 10^{-3} .
$$

$\mathrm{LHCb}$ have more recently performed a similar extraction of $\mathrm{BR}(b \rightarrow \psi(2 S))$ from their data, and have obtained

$$
\mathrm{BR}(b \rightarrow \psi(2 S))^{\mathrm{LHCb}}=(2.73 \pm 0.06 \pm 0.16 \pm 0.24) \times 10^{-3},
$$

fully compatible with the CMS determination. 
When these new branching ratios are used in the FONLL prediction, instead of the $(4.8 \pm 2.4) \times 10^{-3}$ quoted above, the results are, essentially by construction of course, in full agreement with the LHC measurements, as shown in figure 7. On the other hand, the older CDF data now appear to stand out, albeit being potentially marginally compatible with the LHC ones within the respective experimental uncertainties. Since the theoretical predictions for this ratio are largely insensitive to the specific experimental setup, be it the center of mass energy (Tevatron or LHC) or even the rapidity acceptance cuts, the discrepancy is likely of experimental origin.

\section{Open charm and bottom production from inclusive semileptonic de- cays}

In a fashion similar to that employed to describe $J / \psi$ and $\psi(2 S)$ production from $b$-hadron decays, one can describe the production of a lepton $(e$ or $\mu)$ originating from the electroweak decay of a charm or a bottom hadron. As before, the decay spectrum and the branching fraction can be extracted from experimental measurements. This approach has been used with FONLL for the first time in [36], where the prediction for the production rate of electrons from charm and bottom in $p p$ collisions at RHIC was calculated. The results were eventually found in good agreement with measurements by the PHENIX [69] and the STAR [70] collaborations.

Three separate processes contribute to the final yield of leptons $\ell$ from a heavy hadron: $H_{c} \rightarrow \ell, H_{b} \rightarrow \ell$, and the secondary decay $H_{b} \rightarrow H_{c} \rightarrow \ell$. The first contributes mainly at small transverse momentum $\left(p_{T}<5-10 \mathrm{GeV}\right)$, the second dominates at larger $p_{T}$, while the secondary decay is largely negligible (see e.g. figure 3 of [36]). All three processes have been modeled for [36], and we use here exactly the same setup.

Figure 8 shows the cross section predicted by FONLL for the sum of the three processes, as they are largely indistinguishable experimentally. Two NLO-level predictions are also shown. Both make use of the fixed-order heavy quark production NLO calculation for hadronic collisions. In one case (the upper band, blue in colour display, and labeled simply 'NLO') the same non-perturbative fragmentation functions also employed in the FONLL case are used. In the case of the magenta dashed curve instead, labeled 'NLO with NLO NP fit', the non-perturbative fragmentation functions have been fitted to $e^{+} e^{-}$LEP data for $B$ and $D^{*}$ production using a fixed next-to-leading order calculation. The FONLL and the 'NLO' predictions differ significantly at large $p_{T}$ when the same non-perturbative fragmentation functions are used, a consequence of the resummation effects included in FONLL . This difference is however largely compensated - as expected - by the adjustment of the non-perturbative contribution, and one can see that the magenta dashed curve 'NLO with NLO NP fit' is indeed much closer to the FONLL prediction. It should be noted, however, that this prediction employing the NLO fit to LEP data is expected to be valid only in a limited region, where transverse momenta are comparable to the scale $\mu$ at which the fit has been performed $\left(\mu=M_{Z} \simeq 90 \mathrm{GeV}\right)$.

Data for muon production are available from ATLAS [71] up to very large transverse momentum, and one can see in figure 8 how they seem to be better described by the 

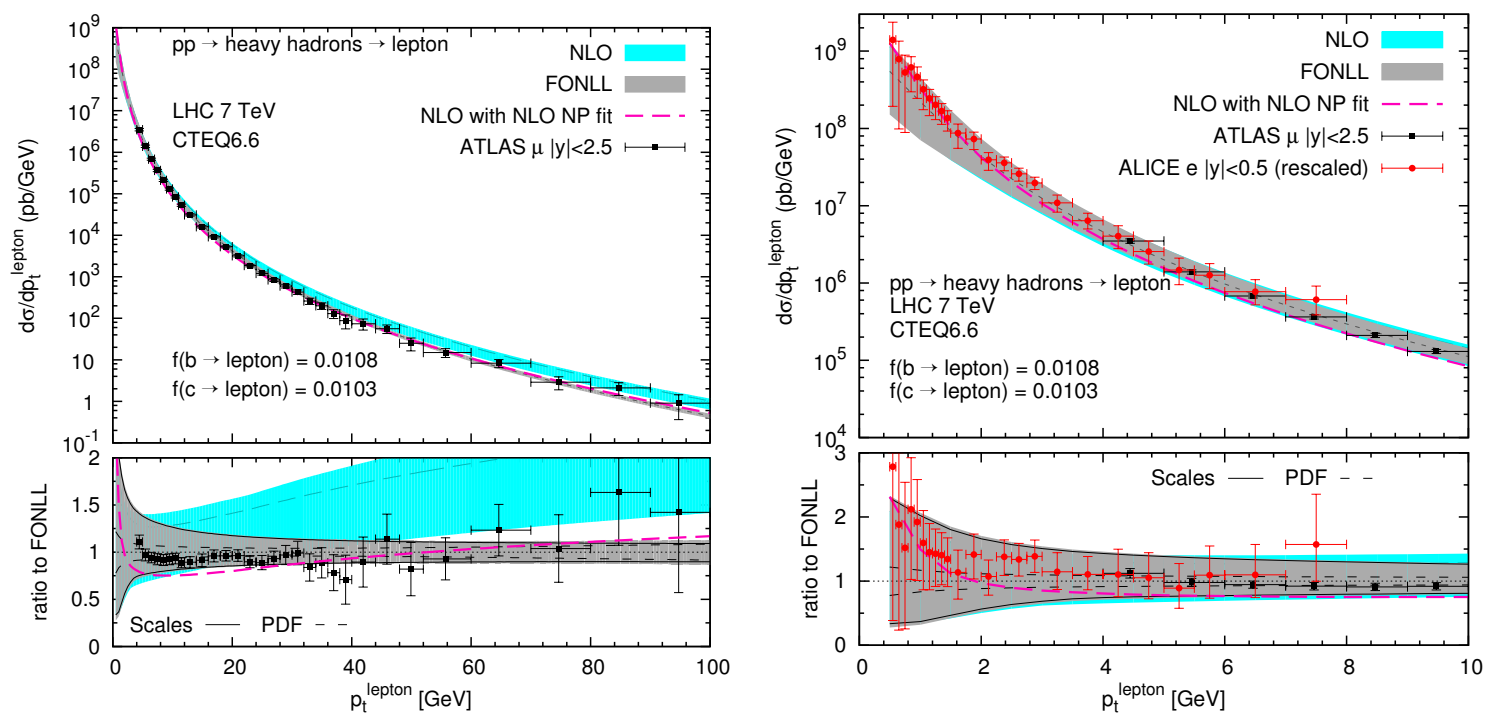

Figure 8. Left plot: transverse momentum distribution of leptons from heavy hadrons in the central rapidity region $|y|<2.5$. The curves represent the sum of the three processes $H_{c} \rightarrow \ell$, $H_{b} \rightarrow \ell$ and $H_{b} \rightarrow H_{c} \rightarrow \ell$. No PDF uncertainties are included in the NLO band. Data from [71] are also shown. Note that both muon charge states are included. Right plot: blow-up of the left one in the $p_{t}<10 \mathrm{GeV}$ region. Electron data from [74], rescaled by a ( $p_{t}$-dependent) factor to account for the different rapidity range and multiplied by two to account for the sum over both charge states, are also shown in this plot.

resummed FONLL prediction than by the fixed order one. Comparing to 'NLO with NLO NP fit' rather than to 'NLO' may appear to largely wash out the edge of FONLL at large $p_{T}$, but the agreement with the data seems to deteriorate at moderate $p_{T}$, as it may be expected from the use of non-perturbative fragmentation functions fitted with a fixed order calculation and at the much larger scale given by the $Z$ mass. In fact, the good agreement with the 'NLO with NLO NP fit' is obtained by trading away the correct description of multiple quasi-colliner emissions via resummation in exchange of their effective inclusion in the non-perturbative fragmentation functions fitted to the NLO calculation. While theoretically less ambitious, the latter procedure is also bound to fail when scales much different than $M_{Z}$ are probed.

The ALICE Collaboration has also produced comparisons of distributions of leptons from heavy hadrons with FONLL predictions, i.e. muons in the forward rapidity region $2.5<y<4$ (see for instance figure 3 of [72] and figure 1 of [73]) and electrons in the central region $|y|<0.5$ (see figure 11 of [74]). The overall agreement is similar to the one with the ATLAS data shown in figure 8. In particular, the right plot of figure 8, which is a blow-up in the $p_{t}<10 \mathrm{GeV}$ region of the left plot of the same figure, shows how the ALICE electron data allow one to extend the comparison with FONLL down to very low transverse momentum.

CMS also published results for transverse momentum and rapidity distributions for muons from $b$-hadrons [75]. The plots in figure 9 compare their results with FONLL and 

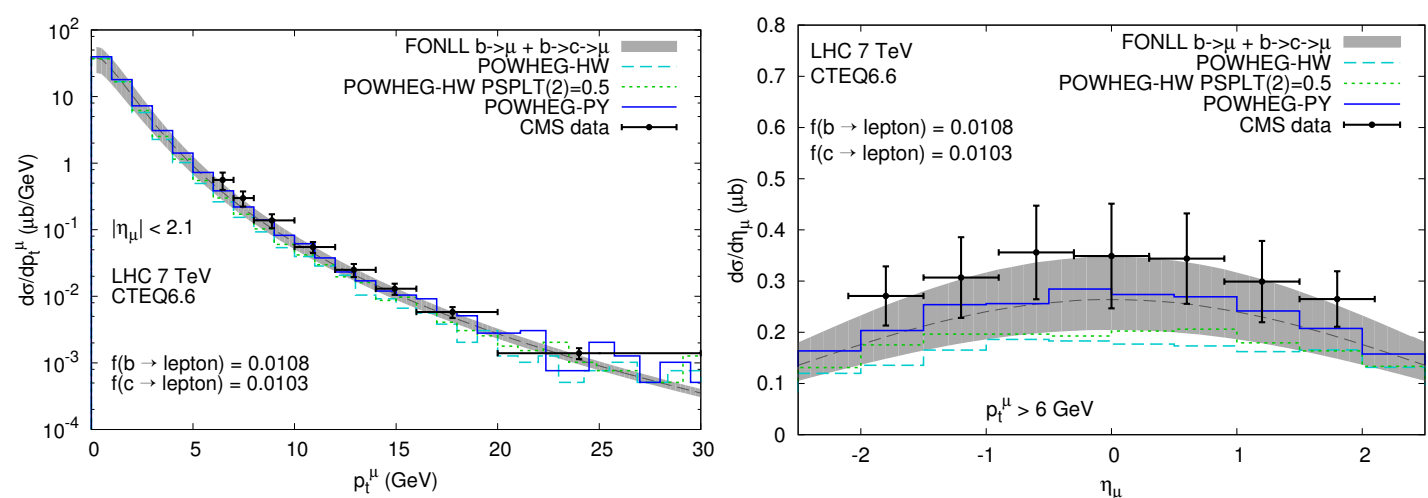

Figure 9. CMS data for muon production from $b$-hadrons decays compared to FONLL and POWHEG predictions. Note that both charge states (i.e. muons from both $b$ and $\bar{b}$ ) are included.

\begin{tabular}{|l|l|l|l|l|}
\hline Expt & Observable $\left(p_{T}\right.$ in $\left.\mathrm{GeV}\right)$ & $\sigma^{\text {exp }}$ & $\sigma^{\text {FONLL }}$ & Comments \\
\hline 1: LHCb [57] & $\sigma\left(H_{b}, 2 \leq \eta \leq 6\right)$ & $89.6 \pm 16.8 \mu \mathrm{b}$ & $70.8_{-24.4}^{+33.3} \mu \mathrm{b}$ & average $b+\bar{b}$ \\
\hline 2: LHCb [58] & $\sigma\left(B^{ \pm}, p_{T}<40,2<y<4.5\right)$ & $41.4 \pm 3.4 \mu \mathrm{b}$ & $40.1_{-14.5}^{+19.0} \mu \mathrm{b}$ & $f\left(b \rightarrow B^{-}\right)=0.403$ \\
\hline 3: CMS [56] & $\sigma\left(B^{0}, p_{T}^{B}>5,\left|y^{B}\right|<2.2\right)$ & $33.2 \pm 4.3 \mu \mathrm{b}$ & $25.5_{-7.1}^{+10.5} \mu \mathrm{b}$ & $f\left(b \rightarrow B^{0}\right)=0.403$ \\
\hline 4: CMS [55] & $\sigma\left(B^{+}, p_{T}^{B}>5,\left|y^{B}\right|<2.4\right)$ & $28.1 \pm 4.4 \mu \mathrm{b}$ & $27.2_{-7.5}^{+11.2} \mu \mathrm{b}$ & $f\left(b \rightarrow B^{-}\right)=0.403$ \\
\hline 5: CMS [59] & $\sigma\left(B_{s}^{0}, 8<p_{T}^{B}<50,\left|y^{B}\right|<2.4\right)$ & $6.9 \pm 0.8 \mathrm{nb}$ & $4.5_{-1.9}^{+2.3} \mathrm{nb}$ & $f\left(b \rightarrow B_{s}^{0}\right)=0.11$ \\
& $\times \mathrm{BR}\left(B_{s}^{0} \rightarrow J / \psi \phi\right)$ & & $\begin{array}{l}\text { (includes BR } \\
\text { uncertainty })\end{array}$ & $\begin{array}{c}\mathrm{BR}\left(B_{s}^{0} \rightarrow J / \psi \phi\right)= \\
(1.4 \pm 0.5) \times 10^{-3}\end{array}$ \\
\hline 6: LHCb [65] & $\sigma\left(H_{b} \rightarrow J / \psi, p_{T}^{\psi}<14,2<y_{\psi}<4.5\right)$ & $1.14 \pm 0.16 \mu \mathrm{b}$ & $1.16_{-0.42}^{+0.55} \mu \mathrm{b}$ & $\mathrm{BR}(b \rightarrow J / \psi)=0.0116$ \\
\hline 7: ALICE [67] & $\sigma\left(H_{b} \rightarrow J / \psi, p_{T}^{\psi}>1.3,\left|y_{\psi}\right|<0.9\right)$ & $1.26 \pm 0.16 \mu \mathrm{b}$ & $1.33_{-0.48}^{+0.59} \mu \mathrm{b}$ & $\mathrm{BR}(b \rightarrow J / \psi)=0.0116$ \\
\hline 8: CMS [75] & $\sigma\left(H_{b} \rightarrow \mu, p_{T}^{\mu}>6,\left|y^{\mu}\right|<2.1\right)$ & $1.32 \pm 0.34 \mu \mathrm{b}$ & $0.855_{-0.19}^{+0.28} \mu \mathrm{b}$ & $\begin{array}{l}\mathrm{BR}(b \rightarrow \ell)=0.0108 \\
\mathrm{BR}(b \rightarrow c \rightarrow \ell)=0.096\end{array}$ \\
\hline
\end{tabular}

Table 1. Summary of various cross-section measurements, compared against the FONLL predictions. The numbers labeling each measurement refer to the entries in figure 10.

POWHEG predictions, and are found to be compatible within uncertainties. From their measurements CMS extract a total visible cross section for muons from $b$-hadron decays,

$$
\sigma^{\mathrm{CMS}}\left(p p \rightarrow H_{b}+X \rightarrow \mu+X^{\prime}, p_{T}^{\mu}>6 \mathrm{GeV},\left|y^{\mu}\right|<2.1\right)=1.32 \pm 0.01 \pm 0.3 \pm 0.15 \mu b
$$

to be compared with the FONLL prediction (which uses the branching ratios $\mathrm{BR}(b \rightarrow \ell)=$ 0.0108 and $\operatorname{BR}(b \rightarrow c \rightarrow \ell)=0.096[60])$

$$
\sigma^{\text {FONLL }}\left(p p \rightarrow H_{b}+X \rightarrow \mu+X^{\prime}, p_{T}^{\mu}>6 \mathrm{GeV},\left|y^{\mu}\right|<2.1\right)=0.855_{-0.19}^{+0.28} \mu b
$$

Note that both charge states are included in these results.

\section{Conclusions}

We have presented theoretical predictions for heavy quark cross sections for various observable final states within realistic acceptance cuts. In a number of cases, we compared the 


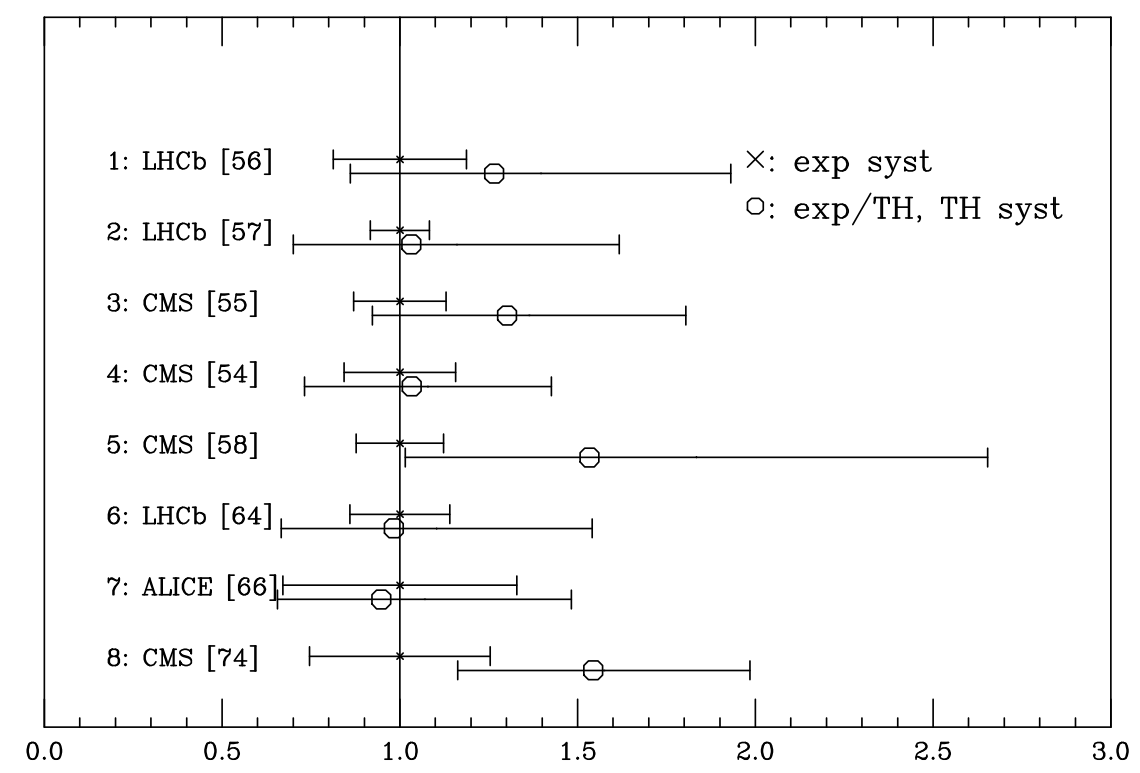

Figure 10. Graphical display of the cross section results listed in table 1. We show the ratios of experimental data over the FONLL calculations, with the sizes of experimental and theoretical systematics. The numbers labeling each measurement refer to the entries in table 1.

predictions of FONLL, MC@NLO and POWHEG with HERWIG and/or PYTHIA. These predictions are also compared directly with experimental measurements at the LHC, and this paper is meant to provide a detailed description and a record of these theoretical results.

The curves presented in this article, as well as others directly delivered to the experimental collaborations, were mostly obtained before any data were available. They have not in the least been influenced by the LHC measurements, and are a direct extension to LHC energy of a framework previously built and validated using LEP and Tevatron data.

One can note that, while FONLL, MC@NLO and POWHEG-based predictions generally agree with each other in the moderate transverse momentum regions, some differences can be observed at large $p_{T}$. The small- $p_{T}$ agreement is expected, as by construction all three frameworks must return the NLO cross section when integrated over the whole phase space, and this result will be dominated by the small- $p_{T}$ region. At large $p_{T}$, instead, differences can arise. Part of them may be due to the Monte Carlo based approaches only resumming the quasi-collinear logarithms to 'almost' leading log accuracy rather than next-to-leading one, but this is expected to be a minor effect. More importantly, nonperturbative fragmentation contributions that had been adjusted to data in PYTHIA and HERWIG are likely not fully appropriate to be used in a matched context where hard gluon radiation to next-to-leading order level is also included. These non-perturbative parametrisations should therefore be re-adjusted to $e^{+} e^{-}$data within the same MC@NLO and POWHEG frameworks that are then going be used to produce predictions for hadronic collisions, analogously to what is done with FONLL. Lack of an appropriate tuning of the non-perturbative hadronization of heavy quarks could provide an explanation for the discrepancy between the fragmentation function of $D^{*}$ mesons inside jets, measured in ref. [76], and the theoretical predictions, based on NLO PSMCs, shown in that paper. 
The agreement between the LHC experimental data we discussed and theoretical predictions is otherwise generally very good. We summarize the various cross sections reported in this paper in table 1 and in figure 10. The comparison of theory and data at small $p_{T}$, as well as at large rapidity, does not point to the existence of a new dynamical regime for the production of heavy quarks (e.g. one dominated by small- $x$ effects), beyond what can be accounted for by fixed-order NLO calculations. The measurements at large transverse momentum, furthermore, seem to favour a description based on FONLL, underscoring the importance of resumming large logarithms of $p_{T}^{Q} / m_{Q}$.

Other sets of measurements not discussed in this paper, such as the $E_{T}$ spectrum and angular correlations of jets containing $b$ quarks [77, 78], are consistent with these findings. Some discrepancies with MC@NLO, on the other hand, have been reported recently in a study of angular correlations between $b$-hadron pairs [79].

It is important to consider that, while the overall theoretical systematics is typically large, most of it is highly correlated in different measurements and in different kinematic regions. This is certainly the case of mass, fragmentation and PDF systematics, but it is also true of the scale systematics. When these correlations are taken into account, the agreement with the experimental data is even more remarkable, particularly considering the efforts that were required, during the first 10 years of measurements at the Tevatron, to reach such a level of consistency.

\section{Acknowledgments}

We acknowledge numerous interactions with many experimental colleagues who, before and during their analyses, suggested the appropriate final states and acceptance cuts for the production of these predictions. In particular, we wish to thank Vincenzo Chiochia, Zaida Conesa del Valle, Andrea Dainese, Biagio Di Micco, Guenther Dissertori, Daniel Froidevaux, Leonid Gladilin, Jibo He, Vato Kartvelishvili, Aafke Kraan, Silvia Masciocchi, Rolf Oldeman, Fabrizio Palla, Gabriella Pasztor, Fabrizio Petrucci, Patrick Robbe, Giovanni Sabatino, Michael Schmelling, Enrico Scomparin, Sheldon Stone. We also thank the Galileo Galilei Institute for Theoretical Physics for the hospitality and the INFN for partial support while part of this work was performed.

Open Access. This article is distributed under the terms of the Creative Commons Attribution License which permits any use, distribution and reproduction in any medium, provided the original author(s) and source are credited.

\section{References}

[1] CDF collaboration, F. Abe et al., A Measurement of the $B$ meson and $b$ quark cross-sections at $\sqrt{s}=1.8 \mathrm{TeV}$ using the exclusive decay $B^{ \pm} \rightarrow J / \psi K^{ \pm}$, Phys. Rev. Lett. 68 (1992) 3403 [INSPIRE].

[2] CDF collaboration, D. Acosta et al., Measurement of the ratio of $b$ quark production cross sections in $\bar{p} p$ collisions at $\sqrt{s}=630 \mathrm{GeV}$ and $\sqrt{s}=1800 \mathrm{GeV}$, Phys. Rev. D 66 (2002) 032002 [hep-ex/0206019] [INSPIRE]. 
[3] CDF collaboration, F. Abe et al., Inclusive $J / \psi, \psi(2 S)$ and $b$ quark production in $\bar{p} p$ collisions at $\sqrt{s}=1.8$ TeV, Phys. Rev. Lett. 69 (1992) 3704 [InSPIRE].

[4] CDF collaboration, F. Abe et al., Measurement of the bottom quark production cross-section using semileptonic decay electrons in p $\bar{p}$ collisions at $\sqrt{s}=1.8 \mathrm{Te}$, Phys. Rev. Lett. 71 (1993) 500 [INSPIRE].

[5] CDF collaboration, F. Abe et al., Measurement of bottom quark production in 1.8 TeV p $\bar{p}$ collisions using semileptonic decay muons, Phys. Rev. Lett. 71 (1993) 2396 [INSPIRE].

[6] CDF collaboration, F. Abe et al., Measurement of the B meson differential cross-section, $d \sigma / d p_{T}$, in $p \bar{p}$ collisions at $\sqrt{s}=1.8$ TeV, Phys. Rev. Lett. 75 (1995) 1451 [hep-ex/9503013] [INSPIRE].

[7] CDF collaboration, D. Acosta et al., Measurement of the $B^{+}$total cross section and $B^{+}$ differential cross section $d \sigma / d p_{T}$ in $p \bar{p}$ collisions at $\sqrt{s}=1.8 \mathrm{TeV}$, Phys. Rev. D 65 (2002) 052005 [hep-ph/0111359] [InSPIRE].

[8] D0 collaboration, S. Abachi et al., Inclusive $\mu$ and $B$ quark production cross-sections in $p \bar{p}$ collisions at $\sqrt{s}=1.8$ TeV, Phys. Rev. Lett. 74 (1995) 3548 [INSPIRE].

[9] D0 collaboration, B. Abbott et al., The $b \bar{b}$ production cross section and angular correlations in $p \bar{p}$ collisions at $\sqrt{s}=1.8$ TeV, Phys. Lett. B 487 (2000) 264 [hep-ex/9905024] [INSPIRE].

[10] D0 collaboration, B. Abbott et al., Small angle muon and bottom quark production in $p \bar{p}$ collisions at $\sqrt{s}=1.8$ TeV, Phys. Rev. Lett. 84 (2000) 5478 [hep-ex/9907029] [INSPIRE].

[11] M. Cacciari, Rise and fall of the bottom quark production excess, hep-ph/0407187 [INSPIRE].

[12] M.L. Mangano, The saga of bottom production in $p \bar{p}$ collisions, AIP Conf. Proc. 753 (2005) 247 [hep-ph/0411020] [INSPIRE].

[13] CDF collaboration, D. Acosta et al., Measurement of the $J / \psi$ meson and b-hadron

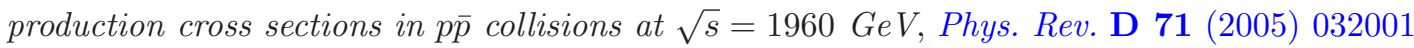
[hep-ex/0412071] [INSPIRE].

[14] CDF collaboration, A. Abulencia et al., Measurement of the $B^{+}$production cross-section in $p \bar{p}$ collisions at $\sqrt{s}=1960$ GeV, Phys. Rev. D 75 (2007) 012010 [hep-ex/0612015] [INSPIRE].

[15] CDF collaboration, T. Aaltonen et al., Measurement of correlated $b^{-} \bar{b}$ production in $p^{-} \bar{p}$ collisions at $\sqrt{s}=1960$ GeV, Phys. Rev. D 77 (2008) 072004 [arXiv:0710.1895] [INSPIRE].

[16] M. Cacciari, M. Greco and P. Nason, The $p_{T}$ spectrum in heavy flavor hadroproduction, JHEP 05 (1998) 007 [hep-ph/9803400] [INSPIRE].

[17] M. Cacciari, S. Frixione and P. Nason, The $p_{T}$ spectrum in heavy flavor photoproduction, JHEP 03 (2001) 006 [hep-ph/0102134] [INSPIRE].

[18] M. Cacciari and P. Nason, Is there a significant excess in bottom hadroproduction at the Tevatron?, Phys. Rev. Lett. 89 (2002) 122003 [hep-ph/0204025] [INSPIRE].

[19] M. Cacciari, S. Frixione, M. Mangano, P. Nason and G. Ridolfi, QCD analysis of first $b$ cross-section data at $1.96 \mathrm{TeV}$, JHEP 07 (2004) 033 [hep-ph/0312132] [INSPIRE].

[20] S. Frixione and B.R. Webber, Matching NLO QCD computations and parton shower simulations, JHEP 06 (2002) 029 [hep-ph/0204244] [INSPIRE].

[21] S. Frixione, P. Nason and B.R. Webber, Matching NLO QCD and parton showers in heavy flavor production, JHEP 08 (2003) 007 [hep-ph/0305252] [INSPIRE].

[22] P. Nason, A New method for combining NLO QCD with shower Monte Carlo algorithms, JHEP 11 (2004) 040 [hep-ph/0409146] [INSPIRE]. 
[23] S. Frixione, P. Nason and G. Ridolfi, A positive-weight next-to-leading-order Monte Carlo for heavy flavour hadroproduction, JHEP 09 (2007) 126 [arXiv:0707.3088] [INSPIRE].

[24] G. Marchesini et al., HERWIG: A Monte Carlo event generator for simulating hadron emission reactions with interfering gluons. Version 5.1- - April 1991, Comput. Phys. Commun. 67 (1992) 465 [InSPIRE].

[25] G. Corcella et al., HERWIG 6: an event generator for hadron emission reactions with interfering gluons (including supersymmetric processes), JHEP 01 (2001) 010 [hep-ph/0011363] [INSPIRE].

[26] G. Corcella et al., HERWIG 6.5 release note, hep-ph/0210213 [INSPIRE].

[27] T. Sjöstrand, S. Mrenna and P.Z. Skands, PYTHIA 6.4 physics and manual, JHEP 05 (2006) 026 [hep-ph/0603175] [INSPIRE].

[28] B. Kniehl, G. Kramer, I. Schienbein and H. Spiesberger, Inclusive B-meson production at the LHC in the GM-VFN scheme, Phys. Rev. D 84 (2011) 094026 [arXiv:1109.2472] [INSPIRE].

[29] B. Kniehl, G. Kramer, I. Schienbein and H. Spiesberger, Inclusive charmed-meson production at the CERN LHC, Eur. Phys. J. C 72 (2012) 2082 [arXiv:1202.0439] [InSPIRE].

[30] P. Nason, S. Dawson and R.K. Ellis, The total cross-section for the production of heavy quarks in hadronic collisions, Nucl. Phys. B 303 (1988) 607 [InSPIRE].

[31] P. Nason, S. Dawson and R.K. Ellis, The one particle inclusive differential cross-section for heavy quark production in hadronic collisions, Nucl. Phys. B 327 (1989) 49 [Erratum ibid. B 335 (1990) 260] [INSPIRE].

[32] W. Beenakker, H. Kuijf, W. van Neerven and J. Smith, QCD corrections to heavy quark production in pp collisions, Phys. Rev. D 40 (1989) 54 [INSPIRE].

[33] M. Cacciari and M. Greco, Large $p_{T}$ hadroproduction of heavy quarks, Nucl. Phys. B 421 (1994) 530 [hep-ph/9311260] [InSPIRE].

[34] M. Cacciari, P. Nason and C. Oleari, A study of heavy flavored meson fragmentation functions in $e^{+} e^{-}$annihilation, JHEP 04 (2006) 006 [hep-ph/0510032] [INSPIRE].

[35] M. Cacciari and P. Nason, Charm cross-sections for the Tevatron Run II, JHEP 09 (2003) 006 [hep-ph/0306212] [INSPIRE].

[36] M. Cacciari, P. Nason and R. Vogt, QCD predictions for charm and bottom production at RHIC, Phys. Rev. Lett. 95 (2005) 122001 [hep-ph/0502203] [INSPIRE].

[37] FONLL heavy quark production, http://www.lpthe.jussieu.fr/ cacciari/fonll/fonllform.html.

[38] V. Kartvelishvili, A. Likhoded and V. Petrov, On the fragmentation functions of heavy quarks into hadrons, Phys. Lett. B 78 (1978) 615 [INSPIRE].

[39] ALEPH collaboration, A. Heister et al., Study of the fragmentation of b quarks into B mesons at the $Z$ peak, Phys. Lett. B 512 (2001) 30 [hep-ex/0106051] [INSPIRE].

[40] OPAL collaboration, G. Abbiendi et al., Inclusive analysis of the $b$ quark fragmentation function in $Z$ decays at LEP, Eur. Phys. J. C 29 (2003) 463 [hep-ex/0210031] [InSPIRE].

[41] E. Braaten, K.-m. Cheung, S. Fleming and T.C. Yuan, Perturbative QCD fragmentation functions as a model for heavy quark fragmentation, Phys. Rev. D 51 (1995) 4819 [hep-ph/9409316] [INSPIRE].

[42] ALEPH collaboration, R. Barate et al., Study of charm production in $Z$ decays, Eur. Phys. J. C 16 (2000) 597 [hep-ex/9909032] [inSPIRE].

[43] P.M. Nadolsky and Z. Sullivan, PDF uncertainties in WH production at Tevatron, eConf C 010630 (2001) P510 [hep-ph/0110378] [INSPIRE]. 
[44] P.M. Nadolsky et al., Implications of CTEQ global analysis for collider observables, Phys. Rev. D 78 (2008) 013004 [arXiv:0802.0007] [inSPIRE].

[45] M. Bahr et al., HERWIG++ physics and manual, Eur. Phys. J. C 58 (2008) 639 [arXiv: 0803.0883] [INSPIRE].

[46] S. Frixione, F. Stoeckli, P. Torrielli and B.R. Webber, NLO QCD corrections in HERWIG++ with MC@NLO, JHEP 01 (2011) 053 [arXiv: 1010.0568] [INSPIRE].

[47] P. Torrielli and S. Frixione, Matching NLO QCD computations with PYTHIA using MC@NLO,JHEP 04 (2010) 110 [arXiv: 1002.4293] [INSPIRE].

[48] M.L. Mangano, P. Nason and G. Ridolfi, Heavy quark correlations in hadron collisions at next-to-leading order, Nucl. Phys. B 373 (1992) 295 [INSPIRE].

[49] J. Alwall et al., A standard format for Les Houches event files, Comput. Phys. Commun. 176 (2007) 300 [hep-ph/0609017] [InSPIRE].

[50] S. Alioli, P. Nason, C. Oleari and E. Re, A general framework for implementing NLO calculations in shower Monte Carlo programs: the POWHEG BOX, JHEP 06 (2010) 043 [arXiv: 1002.2581] [INSPIRE].

[51] S. Frixione, P. Nason and C. Oleari, Matching NLO QCD computations with parton shower simulations: the POWHEG method, JHEP 11 (2007) 070 [arXiv:0709.2092] [INSPIRE].

[52] ALICE collaboration, B. Abelev et al., Measurement of charm production at central rapidity in proton-proton collisions at $\sqrt{s}=7 \mathrm{TeV}, \mathrm{JHEP} 01$ (2012) 128 [arXiv:1111.1553] [INSPIRE].

[53] ALICE collaboration, B. Abelev et al., Measurement of charm production at central rapidity in proton-proton collisions at $\sqrt{s}=2.76 \mathrm{TeV}, \mathrm{JHEP} 07$ (2012) 191 [arXiv: 1205.4007] [INSPIRE].

[54] ATLAS collaboration, Comparison of $D^{(*)}$ meson production cross sections with FONLL and GM-VFNS predictions, PHYS-PUB-2011-012 (2011).

[55] CMS collaboration, V. Khachatryan et al., Measurement of the $B^{+}$production cross section in pp collisions at $\sqrt{s}=7 \mathrm{TeV}$, Phys. Rev. Lett. 106 (2011) 112001 [arXiv:1101.0131] [INSPIRE].

[56] CMS collaboration, S. Chatrchyan et al., Measurement of the B0 production cross section in pp collisions at $\sqrt{s}=7$ TeV, Phys. Rev. Lett. 106 (2011) 252001 [arXiv:1104.2892] [INSPIRE].

[57] LHCB collaboration, R. Aaij et al., Measurement of $\sigma(p p \rightarrow b \bar{b} X)$ at $\sqrt{s}=7 \mathrm{TeV}$ in the forward region, Phys. Lett. B 694 (2010) 209 [arXiv:1009.2731] [INSPIRE].

[58] LHCB collaboration, R. Aaij et al., Measurement of the $B^{ \pm}$production cross-section in pp collisions at $\sqrt{s}=7 \mathrm{TeV}$, JHEP 04 (2012) 093 [arXiv: 1202.4812] [INSPIRE].

[59] CMS collaboration, S. Chatrchyan et al., Measurement of the strange B meson production cross section with $J / \Psi \phi$ decays in pp collisions at $\sqrt{s}=7 \mathrm{TeV}$,

Phys. Rev. D 84 (2011) 052008 [arXiv: 1106.4048] [inSPIRE].

[60] Particle Data Group collaboration, K. Nakamura et al., Review of particle physics, J. Phys. G 37 (2010) 075021 [InSPIRE].

[61] LHCB collaboration, R. Aaij et al., Measurement of b-hadron production fractions in 7 TeVpp collisions, Phys. Rev. D 85 (2012) 032008 [arXiv:1111.2357] [INSPIRE].

[62] BABAR collaboration, B. Aubert et al., Study of inclusive production of charmonium mesons in B decay, Phys. Rev. D 67 (2003) 032002 [hep-ex/0207097] [InSPIRE]. 
[63] CMS collaboration, V. Khachatryan et al., Prompt and non-prompt $J / \psi$ production in $p p$ collisions at $\sqrt{s}=7$ TeV, Eur. Phys. J. C 71 (2011) 1575 [arXiv:1011.4193] [INSPIRE].

[64] CMS collaboration, S. Chatrchyan et al., $J / \psi$ and $\psi(2 S)$ production in $p p$ collisions at $\sqrt{s}=7 \mathrm{Te}$, JHEP 02 (2012) 011 [arXiv: 1111.1557] [INSPIRE].

[65] LHCB collaboration, R. Aaij et al., Measurement of $J / \psi$ production in pp collisions at $\sqrt{s}=7 \mathrm{TeV}$, Eur. Phys. J. C 71 (2011) 1645 [arXiv:1103.0423] [INSPIRE].

[66] ATLAS collaboration, G. Aad et al., Measurement of the differential cross-sections of inclusive, prompt and non-prompt $J / \psi$ production in proton-proton collisions at $\sqrt{s}=7 \mathrm{TeV}$, Nucl. Phys. B 850 (2011) 387 [arXiv:1104.3038] [INSPIRE].

[67] ALICE collaboration, B. Abelev et al., Measurement of prompt and non-prompt $J / \psi$ production cross sections at mid-rapidity in pp collisions at $\sqrt{s}=7 \mathrm{TeV}$, arXiv:1205.5880 [INSPIRE].

[68] LHCB collaboration, R. Aaij et al., Measurement of $\psi(2 S)$ meson production in pp collisions at $\sqrt{s}=7$ TeV, Eur. Phys. J. C 72 (2012) 2100 [arXiv:1204.1258] [INSPIRE].

[69] PHENIX collaboration, A. Adare et al., Measurement of high- $p_{T}$ single electrons from heavy-flavor decays in $p+p$ collisions at $\sqrt{s}=200$ GeV, Phys. Rev. Lett. 97 (2006) 252002 [hep-ex/0609010] [INSPIRE].

[70] STAR collaboration, B. Abelev et al., Erratum: Transverse momentum and centrality dependence of high-p $p_{t}$ non-photonic electron suppression in $A u+A u$ collisions at $\sqrt{s_{N N}}=200$ GeV, Phys. Rev. Lett. 98 (2007) 192301 [Erratum ibid. 106 (2011) 159902] [nucl-ex/0607012] [INSPIRE].

[71] ATLAS collaboration, G. Aad et al., Measurements of the electron and muon inclusive cross-sections in proton-proton collisions at $\sqrt{s}=7$ TeV with the ATLAS detector, Phys. Lett. B 707 (2012) 438 [arXiv:1109.0525] [INSPIRE].

[72] ALICE collaboration, B. Abelev et al., Heavy flavour decay muon production at forward rapidity in proton-proton collisions at $\sqrt{s}=7$ TeV, Phys. Lett. B 708 (2012) 265 [arXiv: 1201.3791] [INSPIRE].

[73] ALICE collaboration, B. Abelev et al., Production of muons from heavy flavour decays at forward rapidity in $\mathrm{pp}$ and $\mathrm{Pb}-\mathrm{Pb}$ collisions at $\sqrt{s_{N N}}=2.76 \mathrm{TeV}$, arXiv:1205.6443 [INSPIRE].

[74] ALICE collaboration, B. Abelev et al., Measurement of electrons from semileptonic heavy-flavour hadron decays in pp collisions at $\sqrt{s}=7 \mathrm{TeV}$, arXiv:1205.5423 [INSPIRE].

[75] CMS collaboration, V. Khachatryan et al., Inclusive b-hadron production cross section with muons in pp collisions at $\sqrt{s}=7$ TeV, JHEP 03 (2011) 090 [arXiv:1101.3512] [INSPIRE].

[76] ATLAS collaboration, G. Aad et al., Measurement of $D^{* \pm}$ meson production in jets from $p p$ collisions at $\sqrt{s}=7 \mathrm{TeV}$ with the ATLAS detector, Phys. Rev. D 85 (2012) 052005 [arXiv: 1112.4432] [INSPIRE].

[77] ATLAS collaboration, G. Aad et al., Measurement of the inclusive and dijet cross-sections of b-jets in pp collisions at $\sqrt{s}=7$ TeV with the ATLAS detector, Eur. Phys. J. C 71 (2011) 1846 [arXiv:1109.6833] [InSPIRE].

[78] CMS collaboration, S. Chatrchyan et al., Inclusive b-jet production in pp collisions at $\sqrt{s}=7 \mathrm{TeV}$, JHEP 04 (2012) 084 [arXiv: 1202.4617] [INSPIRE].

[79] CMS collaboration, V. Khachatryan et al., Measurement of B $\bar{B}$ angular correlations based on secondary vertex reconstruction at $\sqrt{s}=7$ TeV, JHEP 03 (2011) 136 [arXiv:1102.3194] [INSPIRE]. 